\title{
LXXV. On the principles of the application of analysis to the motion of fluids
}

\section{Rev. J. Challis M.A.}

To cite this article: Rev. J. Challis M.A. (1841) LXXV. On the principles of the application of analysis to the motion of fluids , Philosophical Magazine, 18:119, 477-481, DOI:

$10.1080 / 14786444108650335$

To link to this article: http://dx.doi.org/10.1080/14786444108650335

册 Published online: 01 Jun 2009.

Submit your article to this journal

Џll Article views: 3

Q View related articles $\asymp$

Citing articles: 1 View citing articles 
the effect? Might it not be as reasonably inferred, in the case of light, that, although its transmission does not require the interposition of a pane of glass, yet, that when such a pane is interposed, since the light is not intercepted, there is reason to suppose an active cooperation of the vitreous particles in aid of the radiation? It may be expedient here to advert to the fact, that Prof. Henry has found a metallic plate to interfere with the dynamic induction of one flat helix upon another. I have myself been witness of this result.

74. Does not magnetic or electro-dynamic induction take place as well in vacuo as in pleno? Has the presence of any gas been found to promote or retard that species of reaction? It appears that, agreeably to your experiments, ponderable bodies, when made to intervene, did not enhance the influence in question, while in some of those performed by Henry it was intercepted by them. Does it not follow that ponderable particles may impede, but cannot assist in this process?

75. I am happy to find that, among the opinions which I expressed in my letter to you, although there are several in which you do not concur, there are some which you esteem of importance, though you do not consider yourself justified in extending to them your sanction, being constrained, in the present state of human knowledge, to hold your judgment in suspense. For the present I shall here take leave of this subject, having already so extended my letter as to occupy too much of your valuable time. I am aware that as yet I have not sufficiently studied many of the intricate results of your sagacity, ingenuity and consummate skill in experimental investigations. When I shall have time to make them the subject of the careful consideration which they merit, I may venture to subject your patience to some further trials.

Philadelphia, Jan. 1, 1841.

LXXV. On the Principles of the Application of Analysis to the Motion of Fluids. By the Rev. J. Challis, M.A., Plumian Professor of Astronomy and Experimental Philosophy in the University of Cambridge*.

FOR the sake of simplicity, the following remarks will be restricted to the motion of an incompressible fluid: they may without difficulty be extended to fluid motion in general.

It is well known that one of the fundamental equations of fluid motion is obtained on the principle that the mass of each small element remains the same while its position and form

* Communicated by the Author. 
are varying. If $u, v, w$ be the parts of the velocity at a point whose coordinates are $x, y, z$, resolved in the directions of the axes of rectangular coordinates, the equation thus obtained for an incompressible fluid is,

$$
\frac{d u}{d x}+\frac{d v}{d y}+\frac{d w}{d z}=0 \ldots \ldots
$$

Under this form it is not proper for application to proposed instances of motion, for which purpose it must admit of exact or approximate integration, and therefore consist of partial differential coefficients of a principal variable. The transformation into the required form is made by assuming $u d x$ $+v d y+w d z$ to be an exact differential of a function of $x, y$ and $z$. For if $\phi$ be the function, evidently

and

$$
\begin{aligned}
& u=\frac{d \phi}{d x}, v=\frac{d \phi}{d y}, w= \frac{d \phi}{d z}, \\
& \frac{d^{2} \phi}{d x^{2}}+\frac{d^{2} \phi}{d y^{2}}+\frac{d^{2} \Phi}{d z^{2}}=0 \ldots .
\end{aligned}
$$

No general rule has hitherto been given for determining when it is allowable to assume $u d x+v d y+w d z$ to be an exact differential, nor has it been ascertained to what particular circumstance of the motion this analytical condition refers. This must be considered a defect in the mathematical theory of hydrodynamics.

The equation (A.) may also be arrived at by the following considerations. In whatever manner a mass of fluid is in motion, we may conceive an unlimited number of surfaces described in it, so that each is perpendicular to the direction of the motion at a given time of the particles through which it passes. These surfaces are not necessarily continuous, but must be supposed to consist of continuous parts possessing the general properties of curve surfaces. Hence if, in two of the surfaces separated by an indefinitely small interval, two indefinitely small rectangular areas be taken opposite to each other, so that the lines joining the corresponding angular points are normals to the surfaces, then the directions of motion in the fluid element lying between them will, by a known property of curve surfaces, all pass through two focal lines perpendicular to the directions of the normals, and situated in planes at right angles to each other. The normal velocity will in general vary in passing from one point to another of the intersecting surface, in a manner depending on the arbitrary disturbance of the fluid; but taking it to be uniform tbrough the extent of each of the indefinitely small areas, the continuity of the fluid will be maintained if the same quantity 
of fluid passes the two areas at the same time. On this principle I have obtained the equation (A.) in the Cambridge Philosophical Transactions (vol. v. part ii. p. 182). The mathematical reasoning is too long to be inserted. I have recently obtained the corresponding equation for compressible fluids by a similar process.

The above method of obtaining the equation (A.) has the advantage of making known the condition that is introduced when $u d x+v d y+w d z$ is assumed to be an exact differential of a function of $x, y$, and $z$. For if $\mathrm{V}$ be the velocity at the point $x y z$ distant by $r$ from a focal line, and $\theta, \eta$ be two angles fixing the direction of the normal through that point, we may put $\mathrm{V}=f(\theta, \eta) \times \phi(r)$, the latter factor expressing the law of the variation of $\mathrm{V}$ at a given instant through a small space resulting from the convergence or divergence of the normals. Hence if $\alpha, \beta, \gamma$ be the coordinates of the intersection of the normal with the focal line, we shall have

$$
\begin{aligned}
& u=f(\theta, \eta) \cdot \Phi(r) \cdot \frac{x-\alpha}{r} ; v=f(\theta, \eta) \cdot \Phi(r) \cdot \frac{y-\beta}{r} ; \\
& w=f(\theta, \eta) \cdot \Phi(r) \cdot \frac{z-\gamma}{r} ; \text { and } u d x+v d y+w d z \\
& =f(\theta, \eta) \cdot \Phi(r)\left\{\frac{x-\alpha}{r} d x+\frac{y-\beta}{r} d y+\frac{z-\gamma}{y} d z\right\} .
\end{aligned}
$$

Now since $r^{2}=(x-\alpha)^{2}+(y-\beta)^{2}+(z-\gamma)^{2}$, the above quantity is an exact differential of a function of $x, y$, and $z$, if $\alpha, \beta, \gamma, \theta$, and $\eta$ be constant; that is, if the variation with respect to space be in the direction of the motion. This condition, which has hitherto been unnoticed, is independent of the particular mode in which the fluid is disturbed, and being introduced when $d \Phi$ is substituted for $u d x+v d y+w d z$, must be attended to in all the subsequent calculations in which $\phi$ is involved.

The preceding reasoning fails if the lines of motion be not convergent, or the motion be such as the fluid might take if it were either wholly solid, or consisted of solid disconnected parts: for instance, when the fluid mass revolves about a fixed axis and the velocity is a function of the distance from the axis. In fact, for this instance, $u d x+v d y+w d z$ is not an exact differential.

As it appears from the above reasoning that, in general, at the same time that the equation (A.) is transformed into one consisting of partial differential coefficients of a principal variable, the condition is introduced, that the variation with re- 
spect to space is in the direction of the motion, it follows that the transformed equation and its integral containing arbitrary functions must be immediately applied to the parts of the fluid disturbed in a given and arbitrary manner, and where, consequently, the direction of the motion is known. The velocity, and direction of the velocity, at other parts are to be inferred from the laws of propagation of the motion and variation of the velocity which the integration reveals.

Admitting the extension of the foregoing considerations to a compressible fluid, the following is the manner in which the problem of the resistance to a vibrating sphere requires, on these principles, to be treated. 'The general equation in rectangular coordinates, viz.

$$
\frac{d^{2} \phi}{d t^{2}}=a^{2} \cdot\left(\frac{d^{2} \phi}{d x^{2}}+\frac{d^{2} \phi}{d y^{2}}+\frac{d^{2} \phi}{d \Sigma^{2}}\right),
$$

is first to be transformed into polar coordinates, having the centre of the vibrating sphere for the pole. The polar coordinates being $r$ the distance of the point $x y \approx$ from the centre of the sphere, $\theta$ the angle which $r$ makes with the straight line in which the centre is moving, and $\eta$ the angle which the plane of these two lines makes with a vertical plane, regard also being had to the motion of the sphere, the transformation, effected in the manner adopted by Poisson in Art. 2 of his Memoir in the Connaissance des 'Tems for 183k, leads to the equation

$$
\begin{aligned}
& \frac{d^{2} \cdot r \phi}{d \ell^{2}}=a^{2} \cdot\left\{\frac{d^{2} \cdot r \phi}{d r^{2}}+\frac{1}{r^{2} \sin \theta} \cdot \frac{d \cdot\left(\sin \theta \frac{d \cdot r \phi}{d \theta}\right)}{d \theta}\right. \\
& \left.+\frac{1}{r^{2} \sin ^{2} \theta} \cdot \frac{d^{2} \cdot r \phi}{d \eta^{2}}\right\}
\end{aligned}
$$

which is independent of the motion of the sphere. If now the reasoning be just by which $I$ have argued, that into this equation must be introduced the condition, that the variation with respect to space be in the direction in which the velocity is impressed, it will plainly reduce itseif to

$$
\frac{d^{2} \cdot r \phi}{d t^{2}}=a^{2} \cdot \frac{d^{2} \cdot r \varphi}{d r^{2}}
$$

in which, from what has been already said, $₫$ may contain as a factor an arbitrary function of $\theta$ and $\eta$. This is the equation I have employed in the solution I have given of the problem in the Number of this Journal for December 1840, and by it I have been conducted to the value 2 for the coefficient 
of resistance. I find also that the part of the velocity directed to or from the centre of the sphere varies inversely as the square of the distance, and that in a plane drawn at any instant through the centre of the sphere perpendicular to the direction of its motion, the fluid is stationary. Poisson, by integrating the equation with the term containing the differential coefficient with respect to $\theta$ included, finds the coefficient of resistance to be $1 \frac{1}{2}$, the law of variation of the velocity to be inversely as the cube of the distance, and the velocity of the fluid in the above-mentioned plane and in contact with the sphere, to be half the velocity of the sphere. With these statements I leave the problem to the consideration of mathematicians, not venturing, in a question of so much difficulty, to assert unhesitatingly the correctness of my own views.

As an objection may be raised against the reasoning in my communication in the December Number, because it takes account only of terms involving the first power of the velocity, which, by reason of the small factor $\frac{r}{\lambda}$, may not be of larger magnitude than rejected terms involving the square of the velocity, I take this opportunity of mentioning that, in a paper submitted to the Cambridge Philosophical Society, I have carried the approximation to the next degree, and, as night have been anticipated, I obtain the former result, the pressure depending on the square of the velocity being the same on the preceding as on the following half of the surface of the vibrating sphere. In the same paper I have attempted to give an answer to the query proposed at the end of the abovementioned communication, respecting the motion of a small sphere submitted to the mechanical action of the vibrations of an elastic medium. I find that there will be a motion of vibration of the sphere depending on the first power of the velocity of the vibrating medium, and a permanent motion of translation depending on the second power. This result is important if it serves to explain how the vibrations of the xther (assuming them to be like those of air) may at the same lime produce two different effects, such as light and heat, or light and chemical action.

Cambrisge Observatory, April 13, 1841.

Phil. Mag. S. 3. Vol. 18. No. 119. June 1841. 